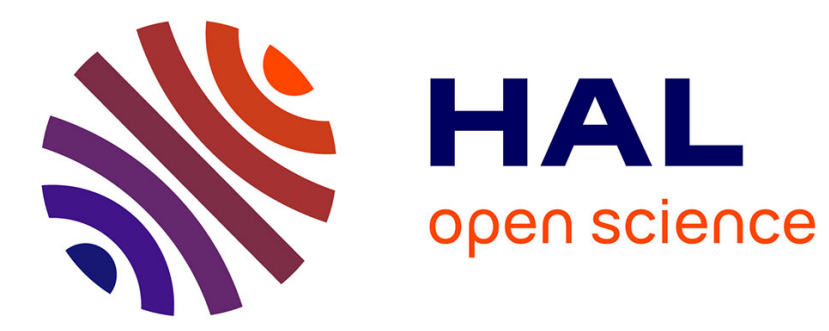

\title{
Josephson junction mixer using an external local oscillator
}

\author{
H. Kanter
}

\section{To cite this version:}

H. Kanter. Josephson junction mixer using an external local oscillator. Revue de Physique Appliquée, 1974, 9 (1), pp.255-262. 10.1051/rphysap:0197400901025500 . jpa-00243749

\section{HAL Id: jpa-00243749 https://hal.science/jpa-00243749}

Submitted on 1 Jan 1974

HAL is a multi-disciplinary open access archive for the deposit and dissemination of scientific research documents, whether they are published or not. The documents may come from teaching and research institutions in France or abroad, or from public or private research centers.
L'archive ouverte pluridisciplinaire HAL, est destinée au dépôt et à la diffusion de documents scientifiques de niveau recherche, publiés ou non, émanant des établissements d'enseignement et de recherche français ou étrangers, des laboratoires publics ou privés. 


\title{
JOSEPHSON JUNCTION MIXER USING AN EXTERNAL LOCAL OSCILLATOR (*)
}

\author{
H. KANTER \\ Electronic Research Laboratory, The Aerospace Corporation \\ Los Angeles, California 90009, USA
}

\begin{abstract}
Résumé. - Nous décrivons des expériences de conversion de fréquence de $95 \mathrm{GHz}$ à $140 \mathrm{MHz}$ à l'aide de contacts en niobium. Le rendement de conversion est inférieur à celui prédit par le modèle de jonction à couplage faible fortement amorti. La différence est due probablement au mélange avec les fréquences harmoniques de l'oscillateur local. La température de bruit la plus basse mesurée est $T \simeq 100 \mathrm{~K}$; elle est donc du même orḍre de grandeur que celle d'une diode à barrière de Schottky à basse température.
\end{abstract}

\begin{abstract}
Mixing experiments on niobium point contacts are reported for conversion from $95 \mathrm{GHz}$ to $140 \mathrm{MHz}$. The conversion efficiency is less than predicted by the highly damped weak link model. The discrepancy is presumably due to mixing with higher harmonics of the local oscillator. The lowest noise temperature measured was $T \approx 100 \mathrm{~K}$ which is of the same order as recently reported for cooled Schottky-barrier diodes.
\end{abstract}

1. Introduction. - Recently several authors, employing analytical and numerical methods, studied the use of Josephson junctions as microwave mixers on the basis of the highly damped junction model [1], [2]. These studies indicate that good performance should be realized when use is made of their nonlinear properties along with an external local oscillator. Accordingly, at frequencies sufficiently below the cutoff due to supercurrent inductance and shunting resistance, the device should function approximately as a broad band switch and, thus, not differ principally from the performance of conventional semiconductor diodes. Furthermore, the model predicts that conversion gain is possible because of the favorable ratio of output to input impedance. In this work the mixing properties of point contact junctions as well as their noise properties are investigated experimentally. In previous experiments point contacts when operated as detectors performed in reasonably close accord with the predictions of the model [3]. Here again the junctions are operated like conventional diodes, that is, they are operated in a waveguide in a broad band fashion; cavities or other frequency selective circuitry are avoided. Since the main goal was to test the junction model no attempt was made to optimize conditions for maximum signal conversion.

The measured conversion efficiencies are lower than the predicted ones. It is believed that the main reason for this is the broadband nature of the device which generates mixing currents with higher harmo-

(*) This work was supported by the US Air Force under Contract \# F 04701-73-C 0074. nics of the local oscillator frequency. Because of the relatively small contact impedance, the contact capacity may be insufficient to short out those currents which consequently dissipate part of the signal energy. (The rich harmonic spectum of the oscillator frequency is demonstrated in mixing with the third harmonic.) For this reason, optimized conversion might require a greater contact capacity or more sophisticated associated circuitry for which the model is not applicable.

Noise temperatures measured on point contacts irradiated with local oscillator power were found to be as low as $T \approx 100 \mathrm{~K}$ which is about the same order as has been recently measured by Weinreb and Kerr [4] on conventional Schottky barrier diodes cooled to $15 \mathrm{~K}$. The measured junction noise is of the order predicted by presently available noise theory [5], [6] although the dependence on junction bias could not be verified.

The experimental results indicate that conversion as well as noise properties of Josephson junctions require more detailed studies before performance limits can be predicted with any confidence. On the basis of these experiments along with the prediction by the model of a frequency cutoff which is approximately the same as that for conventional semiconductor diodes, the performance of superconducting point contacts appears to be comparable to that of semiconductor diodes.

2. Experimental. - The mixing experiments were performed with a setup sketched in figure 1 . The $95 \mathrm{GHz}$ radiation of two klystrons was fed via an $E$-band waveguide into the dewar. One klystron 
served as local oscillator, the other served as the signal source. The klystrons were frequency locked in order to ensure sufficient stability of the $140 \mathrm{MHz}$ difference frequency. Power from each source was calibrated at the mixer to within $2 \mathrm{~dB}$. In the helium bath a $100 \mu$ diameter wire was placed across the full height waveguide for maximum $E$-field interaction. The point was formed nearly in the plane of the waveguide wall with the center conductor of a $1 \mathrm{~mm}$ diameter NbTi stub, which was used to extract the difference frequency signal. The waveguide was terminated with a matched load.

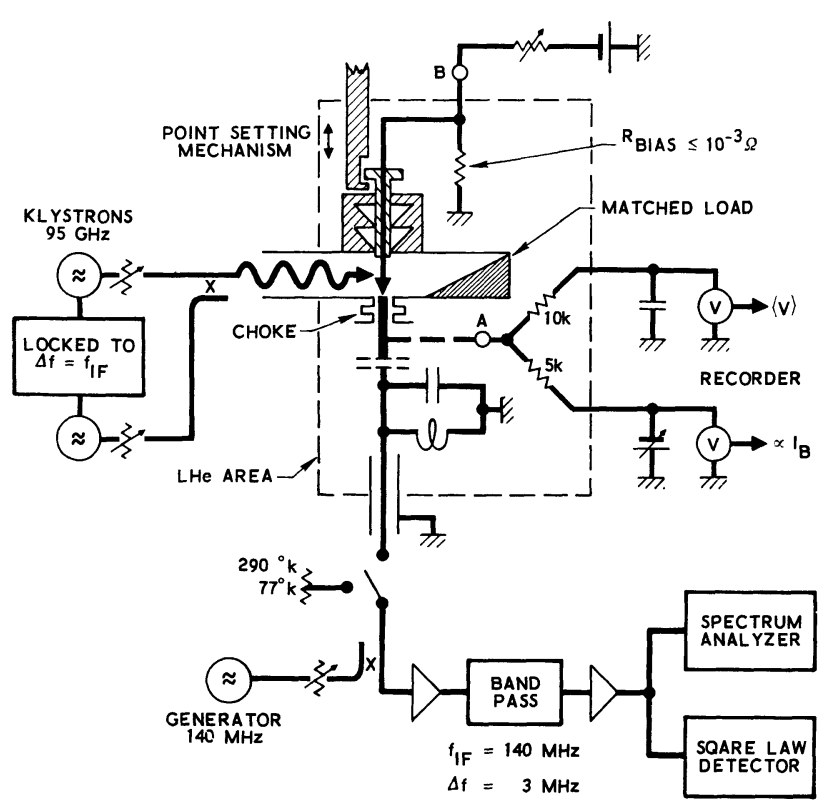

FIG. 1. - Sketch of the experimental setup.

As close to the point as feasible $(5 \mathrm{~mm})$ the stub was connected to a broad band filter $(Q \approx 4)$ through which the circuit for the difference frequency current was completed. Bias was applied by either controlling current or voltage. In the first method a blocking capacitor $(200 \mathrm{pF})$ was inserted between stub and filter and dc-current injected via a $5 \mathrm{k} \Omega$ resistor as shown at point $\mathrm{A}$ in figure 1 . The average voltage could then be measured and $I-V$ characteristics plotted. In the second method no blocking capacitor was used. A dc-potential across the contact was generated by passing a dc-current, at point $B$ in figure 1, through the brass structure between stub and wire which had a resistance of less than $10^{-3} \Omega$. The bias potential was measured, but not the contact current and thus, no $I-V$ characteristic could be plotted. With this bias circuit junction operation was determined entirely by the dynamical response. This method had the advantage of contact bias being independent of local oscillator power.

The signal from the filter was fed via a $50 \Omega$ coaxial line and a directional coupler to the preamplifier. Proper match at the difference frequency was ensured by measuring the relative amplitude, $r$, reflected from the junction with an external $140 \mathrm{MHz}$ signal. After a $3 \mathrm{MHz}$ band pass filter and further amplification the junction output was displayed on a spectrum analyzer and the total power measured with a square law detector. The portion of the setup submerged into the liquid helium was shielded by a lead can. All measurements were performed in a screened room.

The actual measurements proceeded as follows :

When the $I-V$ characteristic was measurable the point contact was set to a zero voltage step current in the range of $10-40 \mu \mathrm{A}$. I- $V$ characteristics were plotted for various local oscillator power levels (Fig. 2a). Extrapolating the straight sections to zero voltage, the step variation with power was obtained (Fig. 2b). Periodic variations of the step for larger power levels were recorded when observable. With application of signal power, the difference signal was observed on the spectrum analyzer together with the signal injected at the difference frequency and reflected by the junction. Both signals were displaced in frequency by less than $1 \mathrm{MHz}$. By manipulating both bias and local oscillator power the maximum signal output for constant signal input power was found under the condition that the reflected amplitude became $r \leqslant 0.1$. Reflection amplitude was measured relative to a shorted input by biasing to zero voltage. The power conversion was obtained by comparing the signal input power with the power required by the $140 \mathrm{MHz}$ generator feeding directly into the amplifier to produce the same signal output.

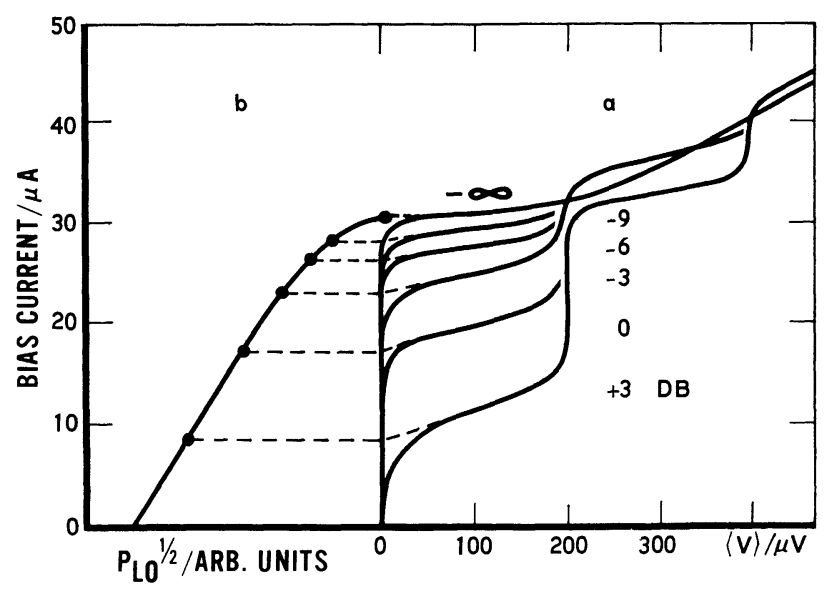

FIG. 2. $-a$ ) Measured $I-V$ characteristics for various oscillator power levels. The parameter is the attenuator setting relative to $10^{-8}$ W. $b$ ) Extrapolated depression of zero voltage step with oscillator amplitude.

When the $I-V$ characteristic could not be measured the point was set by observing the reflected $140 \mathrm{GHz}$ signal. The contact quality could be estimated by the local oscillator power required to generate a change in the reflected amplitude. For a good impedance match contacts operated by the latter method required bias voltages between one-half to one 
times the first step voltage while in the former method bias voltages were lower.

On contacts with reasonable conversion ratios noise measurements were made by measuring the total noise power passing the $3 \mathrm{MHz}$ filter with a square law detector. Usually these measurements could be made with the local oscillator power on and off but the bias had to be changed to maintain the input impedance of $50 \Omega$. For the lowest noise temperatures measured application of local oscillator power generally increased the noise level. Noise temperatures were calibrated with a $50 \Omega$ resistor which was replacing the contact held at ambient, liquid nitrogen and liquid helium temperatures. The amplifier noise contribution was equivalent to a noise temperature between 65 and $70 \mathrm{~K}\left({ }^{1}\right)$. The amplifier noise figure could quickly be checked between measurements by replacing the coaxial line into the dewar with an identical one terminated by a resistor which could be cooled to $77 \mathrm{~K}$ or $4.2 \mathrm{~K}$. Since for all measurements the amplifier input was well matched $(r \leqslant 0.1)$ noise temperatures are believed to be accurate to within $\pm 10 \mathrm{~K}$.

3. Results. - The results on conversion ratio and contact noise for a number of contacts are listed in table $\mathrm{I}$. The measured conversion ratios, $C$ of column 5 of table I established an upper limit near $-20 \mathrm{~dB}$, which to a large part represents the coupling loss through the input network. How the coupling

\section{TABLE I}

Operating values, conversion ratio and noise temperatures for various samples. $\omega_{\mathrm{c}}$ is determined from $I_{\mathrm{c}}$ and $R_{\mathrm{J}}$ as observed from the $I-V$ characteristic or from the periodic variation of $I_{0}$, the zero voltage current step, with oscillator amplitude. The LO power values were generally uncritical. $C$ is the measured conversion ratio, $C_{\text {eff }}$ an intrinsic conversion explained in the text. $T_{0}$ is the noise temperature of a contact with no radiation applied. $\Delta T(\mathrm{LO})$ represents the increase of noise temperature with local oscillator power applied.

\begin{tabular}{|c|c|c|c|c|c|c|c|}
\hline No. & $\begin{array}{c}I_{\mathrm{C}} \\
(\mu \mathrm{A})\end{array}$ & $\omega_{\mathrm{LO} / \omega_{\mathrm{C}}}$ & $\begin{array}{c}\mathrm{LO}^{-8} \text { PWR } \\
10^{-8} \text { Watt }\end{array}$ & $\begin{array}{c}\mathrm{C} \\
(-\mathrm{DB})\end{array}$ & $\begin{array}{c}\mathrm{C}_{\mathrm{eff}} \\
(-\mathrm{DB})\end{array}$ & $\begin{array}{c}\mathrm{T}_{0} \\
{\left[{ }^{\circ} \mathrm{K}\right)}\end{array}$ & $\begin{array}{c}\Delta \mathrm{T}(\mathrm{LO}) \\
{\left[{ }^{\mathrm{K} K}\right]}\end{array}$ \\
\hline 10 & -- & -- & 1.8 & 24 & & 50 & 85 \\
11 & -- & 0.2 & 3.5 & 21 & & 67 & 26 \\
13 & 31 & 0.35 & 0.7 & 21 & 9 & 190 & 200 \\
18 & 45 & 0.28 & 0.7 & 19 & 5 & 157 & 38 \\
20 & -- & -- & 5.6 & 23 & & 137 & 28 \\
\hline
\end{tabular}

(1) The first amplifier stage consisted of a Fairchild FET 0607 MOSFET cooled to liquid nitrogen temperature. loss can be improved is shown in the Appendix (Section 6.1). As expected from the model conversion was not critically dependent on oscillator power : a broad maximum in the dependence of $C$ on local oscillator power occurred for $I_{0} \approx 1 / 2 I_{\mathrm{c}}$. The significance of $C_{\text {eff }}$ (column 6), which is a measure of additional losses, is discussed later. $T_{0}$ is the noise temperature measured for a junction with no local oscillator power applied, while $\Delta T$ (LO) refers to the increase in noise temperature caused by local oscillator power. For correct impedance matching the latter measurement usually required a bias readjustment. The best total noise temperature for mixing was $93 \mathrm{~K}$ (sample \# 11). How the results relate to the predictions of the resistive junction model will be discussed in the following section.

4. Discussion. - 4.1 MoDEL. - The junction model consists of the parallel combination of an ideal superconducting weak link and a resistor, $R_{\mathrm{J}}$. Both are driven by current-sources (Fig. 3). The supercurrent is represented by $I_{\mathrm{J}}=I_{\mathrm{c}} \sin \varphi_{0}^{-1} \int V \mathrm{~d} t$ which follows from Josephson's equations for the limit of contact diameter small compared with the penetration depth of the electromagnetic field. $V$ is the contact voltage, $I_{c}$ the critical current, $\varphi_{0}$ the flux quantum $/ 2 \pi$. The circuit equation then reads

$$
V / R_{\mathrm{J}}+I_{\mathrm{c}} \sin \varphi_{0}^{-1} \int V \mathrm{~d} t=I_{\mathrm{B}}+I_{\mathrm{AC}} \sin \omega t,
$$

which has been solved analytically [7], [8], [9] for $I_{\mathrm{AC}}=0$. The $I-V$ characteristic $\left(I_{\mathrm{B}}\right.$ vs $\left.<V>\right)$ is described by a hyperbola with the apex at

$$
\left(<V>=0, I_{\mathrm{B}}=I_{\mathrm{c}}\right)
$$

and the slope of the asymptote equal to $R_{\mathrm{J}}^{-1}$ (Fig. $4 a$ ).

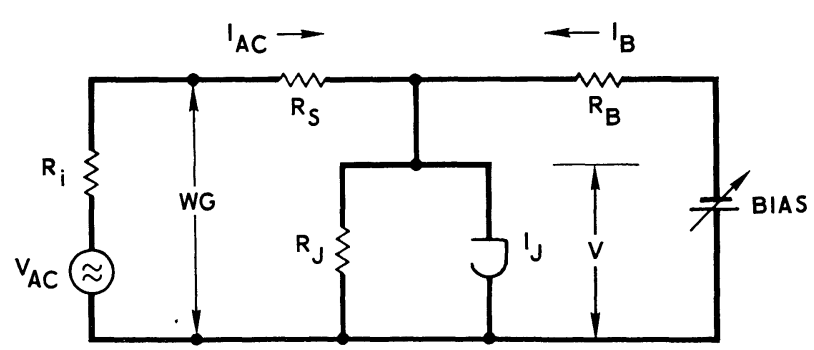

FIg. 3. - Circuit diagram. The source with $V_{\mathrm{AC}}$ and $R_{i}$ represents the waveguide (WG) at the contact wire.

4.2 RESPONSE TO LOCAL OSCILlator. - For arbitrary $I_{\mathrm{AC}}$ only numerical solutions of eq. (1) have been obtained [1], [2], [10], [11]. Qualitatively, the results show that the height of the zero voltage step of the unperturbed $I-V$ characteristic decreases with an increase of $I_{\mathrm{LO}}$ and further steps appear on the characteristic at voltages $V=n \hbar \omega_{\mathrm{LO}} / 2$ e (Fig. $\left.4 b\right)$ where $n$ is an integer. For the following discussion 


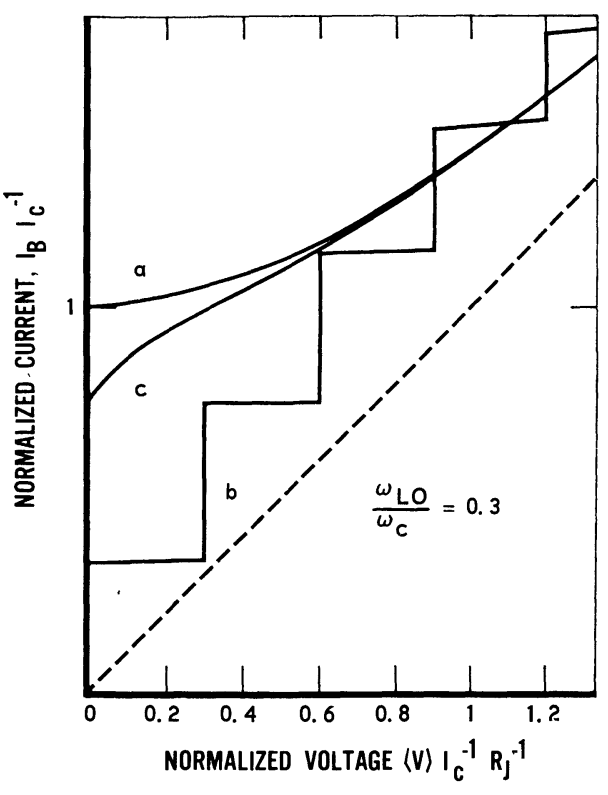

FIG. 4. - Predictions of resistively by-passed junction model : a) Unperturbed $I-V$ characteristic ; b) $I-V$ characteristic with local oscillator of amplitude $I_{\mathrm{LO}} / I_{\mathrm{c}}=1$ at frequency

$$
\omega_{\mathrm{LO}} / \omega_{\mathrm{c}}=0.3
$$

c) Unperturbed $I-V$ characteristic for series inductance $L=10 \hbar / 2 \mathrm{e} I_{\mathrm{c}}$ according to reference [8].

only the region in the vicinity of $\langle V\rangle \ll \hbar \omega_{\text {LO }} / 2 \mathrm{e}$ is of interest.

The variation of the step height $I_{0}$ with $I_{\mathrm{LO}}$ is dependent on the value of $\omega_{\text {LO }}$ relative to

$$
\omega_{\mathrm{c}}=2 \mathrm{e} I_{\mathrm{c}} R_{\mathrm{T}} / \hbar,
$$

the characteristic frequency of the circuit, where $R_{\mathrm{T}}$ is the parallel combination of $R_{\mathrm{J}}$ with $R_{\mathrm{B}}$ and $\left(R_{\mathrm{S}}+R_{i}\right)$. The zero voltage step variations for two frequencies $\omega_{\mathrm{LO}} / \omega_{\mathrm{c}}=0.3$ and $\omega_{\mathrm{LO}} / \omega_{\mathrm{c}}=1$ are shown in figure 5. For $\omega_{\mathrm{LO}} / \omega_{\mathrm{c}} \gtrsim 1$ the step variation is closely described by the ordinary Bessel function of zeroth order, $J_{0}(\xi)$ where $\xi=I_{\mathrm{LO}} / I_{\mathrm{c}}$. The conductance of the junction is essentially determined by $R_{\mathrm{T}}$, and the pair phase is voltage controlled. The

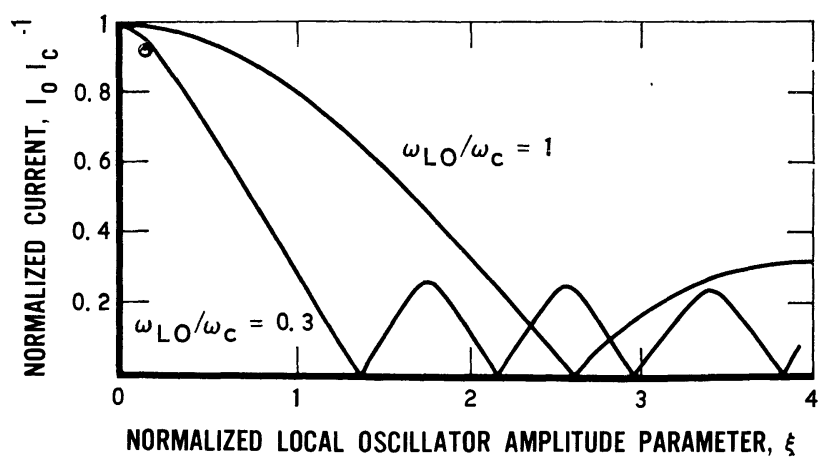

FIG. 5. - Variation of zero voltage step, $I_{0} / I_{\mathrm{c}}$, with local oscillator amplitude, $\xi=I_{\mathrm{LO}} / I_{\mathrm{c}}$ for two normalized frequencies as predicted by model. argument approaches $\xi \rightarrow 2 \mathrm{e} I_{\mathrm{LO}} R_{\mathrm{T}} / \hbar \omega_{\mathrm{LO}}$ and is strongly frequency dependent. This frequency dependence does not exist for $\omega_{\mathrm{LO}} / \omega_{\mathrm{c}} \ll 1$. The junction response is then more properly described by that of a switch, which changes abruptly from infinite to finite conductance once the driving current amplitude exceeds the critical value $I_{c}$. Here the pair current provides the dominant current path in the junction. For $\omega_{\mathrm{LO}} / \omega_{\mathrm{c}} \ll 1$ the zero voltage current step of the $I-V$ characteristic is depressed by $I_{\mathrm{LO}}$, since

$$
I_{0}+I_{\mathrm{LO}} \leqslant I_{\mathrm{c}} \text { for }\langle V\rangle=0 .
$$

For intermediate frequency values a gradual transition occurs for the zero step response. The maximum variation of $I_{0}$ with $I_{\mathrm{LO}}$ is frequency dependent

$$
\Delta I_{0}=S\left(\omega_{\mathrm{LO}}\right) \Delta I_{\mathrm{LO}}
$$

where $S=1$ for $\omega_{\mathrm{LO}} / \omega_{\mathrm{c}} \ll 1$ and $S=0.58 \omega_{\mathrm{LO}} / \omega_{\mathrm{c}}$ for $\omega_{\mathrm{LO}} / \omega_{\mathrm{c}} \gtrsim 1$. $S$ has been numerically determined for various frequencies as shown in figure 6 [1], [2], [10], [11]. For maximum response at $\omega_{\text {Lo }} / \omega_{\mathrm{c}} \ll 1$ it is desirable to have $\omega_{\mathrm{c}}$ maximized by having $R_{\mathrm{S}}+R_{i}$ and $R_{\mathrm{B}} \gg R_{\mathrm{J}}$ (Fig. 3). Since for weak links $2 \mathrm{e} I_{\mathrm{c}} R_{\mathrm{J}}=\Delta_{\mathrm{G}}$ is principally limited by the superconducting gap parameter, $\Delta_{\mathrm{G}}$, the maximum frequency cutoff is material dependent, i. e., $\omega_{\mathrm{c}} / 2 \pi \approx 700 \mathrm{GHz}$ for niobium and $\omega_{\mathrm{c}} / 2 \pi \approx 1000 \mathrm{GHz}$ for $\mathrm{NbN}_{2}$. Thus, for operation at frequencies in excess of $100 \mathrm{GHz}$ with niobium point contacts, loading of the junction by the circuit should remain small.

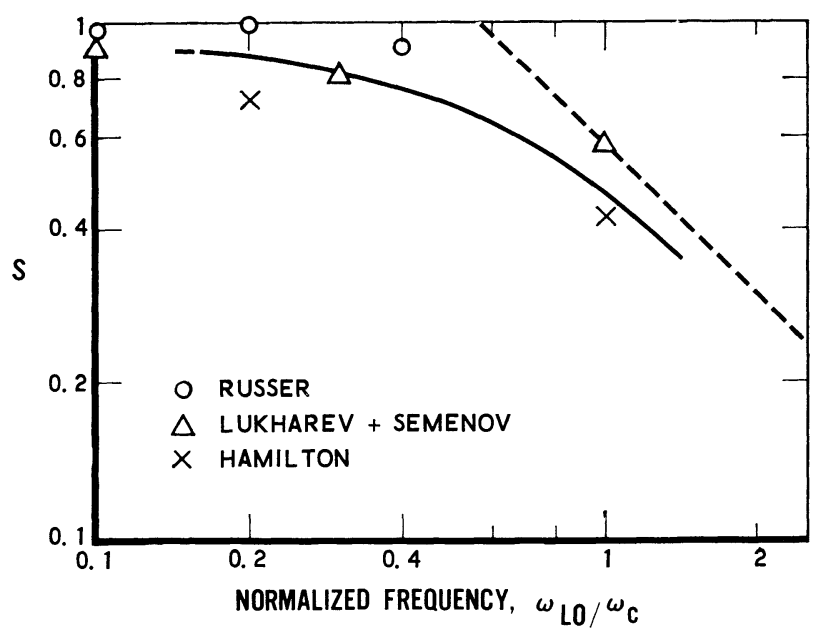

FIG. 6. $-S$-parameter vs frequency. The solid line is due to reference [1], the date points are taken from reference [2], [10] and [11], respectively.

The point contact junctions used in these experiments were less than ideal according to the $\omega_{\mathrm{c}}$ values obtained. We found $0.2 \omega_{\mathrm{c}}(\mathrm{Nb})<\omega_{\mathrm{c}}(\operatorname{Exp})<0.5 \omega_{\mathrm{c}}(\mathrm{Nb})$ for our contacts as determined from $\left(I_{\mathrm{c}} \cdot R_{\mathrm{J}}\right)$ either estimated from the $I-V$ characteristic or from the periodic dependence on local oscillator amplitude of 
the depression to zero of the zero voltage step, $I_{0}$. (Periodic variations beyond the first depression to zero were not observed for all samples which showed good conversion ratios.) Since the actual $\omega_{c}$ of our junctions was considerably reduced below the ideal value for niobium junctions, further loading by the external circuit had to be avoided in order to maintain $S$ values close to one $\left({ }^{2}\right)$.

The requirement for no loading of the junction by the external circuit was met with the experimental arrangement used here, where the thin niobium wire provided the series impedance which in this case is inductive $\left(R_{\mathrm{S}}\right.$ in figure 3 is replaced by $\left.\omega L\right)$. As shown by McCumber [8], by choosing $L \gg \hbar / 2$ e $I_{\mathrm{c}}$ the circuit approaches the current driven behavior for the resistive case as is evident from the $I-V$ characteristic. The deviation from the resistive limit for $\beta=2 \mathrm{e} L I_{\mathrm{c}} / \hbar=10$ is shown in figure 4 , curve $C$. In practice for $\beta \gtrsim 10$ little difference in operation of the circuit from the resistive limit is expected. In our circuit with $L \approx 6 \times 10^{-10} \mathrm{H}$ and $I_{\mathrm{c}} \approx 3 \times 10^{-5}$ A we have $\beta \approx 50$.

In this experiment the waveguide acts as power source with $R_{i}=Z_{0}$ with a value for $Z_{0} \approx 440 \Omega$. A voltage amplitude, $V_{\text {LO }}$, is generated across the wire resulting in a driving current $I_{\mathrm{LO}} \approx V_{\mathrm{LO}} / \omega L$ since $\omega L \gg\left\langle Z_{\mathrm{J}}\right\rangle$ the average contact impedance. $V_{\text {LO }}$ is related to the incoming waveguide power with

$$
P_{\mathrm{IN}}=\frac{\alpha^{2}\left(V_{\mathrm{LO}} \sin \omega_{\mathrm{LO}} t\right)^{2}}{Z_{0}}
$$

where $\alpha$ is dependent on the voltage reflection coefficient introduced by the mismatch of wire and waveguide. (For open termination $\alpha=\alpha_{\max }=2$.) By proper choice of wire diameter to waveguide width the reflection can be made such that $\alpha \gtrsim 1$ in eq. (3). The wire diameter was chosen to give $\alpha \approx 1.4$ and $\omega L \lesssim 400 \Omega$. The requirement $\omega L \gg\left\langle Z_{\mathrm{J}}\right\rangle$ was readily met since $R_{\mathrm{J}} \approx 20 \Omega$ and $\left\langle Z_{\mathrm{J}}\right\rangle<R_{\mathrm{J}}$ because of the relatively large local oscillator amplitude. Auracher and van Duzer [14] have found from computer studies of this junction model for $I_{\mathrm{LO}} / I_{\mathrm{c}}=0.3$ and $\omega_{\mathrm{LO}} / \omega_{\mathrm{c}}=0.4$ the average contact impedance $0<\left\langle Z_{\mathrm{J}}\right\rangle<0.8 R_{\mathrm{J}}$, the actual value depending on bias. (A small region where $\left\langle Z_{\mathrm{J}}\right\rangle<0$ for bias near the first step has been neglected.) Unfortunately, additional reflections in the waveguide structure increased the uncertainty in determining $V_{\text {LO }}$ sufficiently as to preclude systematic measure-

(2) The less-than-ideal $\omega_{\mathrm{c}}$ value of our junctions is believed to be a consequence of our contact preparation. It consisted of filing the flat surface and cutting the thin wire under a shallow angle in order to produce a fine tip before submerging into helium. No further treatment was used. By employing more sophisticated methods of contact preparation other experimenters produced niobium weak links with $\omega_{\mathrm{c}}$ close to the ideal value [12], [13]. ment of $S$ in eq. (2). The observed ratio of junction currents obtained from eq. (2) and (3) gave $1 / 2<S<3 / 2$. We can only conclude that $S$ did not differ greatly from one.

4.3 Mixing OF A SMALl Signal WITH THE LOCAL OSCILlATOR. - The ac current now is

$$
I_{\mathrm{AC}}+I_{\mathrm{LO}} \sin \omega_{\mathrm{LO}} t+I_{\mathrm{S}} \sin \omega t .
$$

For $I_{\mathrm{S}} \ll I_{\text {LO }}$ and $\omega-\omega_{\text {LO }} \ll \omega_{\text {LO }}$

$$
I_{\mathrm{AC}} \rightarrow\left[I_{\mathrm{LO}}+I_{\mathrm{S}} \cos \left(\omega-\omega_{\mathrm{LO}}\right) t\right] \sin \omega_{\mathrm{LO}} t .
$$

The local oscillator amplitude, modulated with the beat signal, is demodulated by the junction. From eq. (2) the ensuing variation of $I_{0}$ is

$$
\Delta I_{0}=S I_{\mathrm{S}} \sin \left(\omega-\omega_{\mathrm{LO}}\right) t
$$

where the bias requirement now is

$$
\hbar\left(\omega-\omega_{\text {LO }}\right) / 2 \text { e } \ll\langle V\rangle \ll \hbar \omega_{\text {LO }} / 2 \text { e } .
$$

Considering the junction as a generator of the difference frequency signal with an internal resistance $R_{\mathrm{D}}$, where $R_{\mathrm{D}}^{-1}=\mathrm{d} I_{\mathrm{B}} / \mathrm{d}\langle V\rangle$, one obtains the maximum power which can be delivered by the junction :

$$
P_{\text {out }}=\left(\Delta I_{0}\right)^{2} \cdot \frac{R_{\mathrm{D}}}{8}
$$

By combining eq. (3), (4) and (5) the conversion ratio of signal power into the difference frequency is obtained :

$$
C=\frac{P_{\text {out }}}{P_{\text {in }}}=\frac{S^{2} \alpha^{2}}{4} \frac{Z_{0} R_{\mathrm{D}}}{(\omega L)^{2}} .
$$

Inserting the proper values and assuming $S=1$ and $\alpha=1.4$ we obtain $C=0.063(-12 \mathrm{~dB})$ which is to be compared with the measured values of table $\mathrm{I}$, labeled $C$. A discrepancy of at least $-7 \mathrm{~dB}$ exists which cannot be entirely accounted for with $S<1$. Conversion losses encountered beyond those predicted by eq. (6) were measured by adapting the following procedure: From the measured variation of $I_{0}$ with local oscillator power (Fig. $2 b$ ) the small signal power requirement for a certain power output could be determined. This value was compared with the actually measured one and the difference in conversion efficiency is labeled $C_{\text {eff }}$ in table $\mathrm{I}\left({ }^{3}\right)$. In this way the uncertainties due to $S$ and the input network were both eliminated. $C_{\text {eff }}$ measures all factors contri-

(3) The small signal requirement was met by operating at power levels near $10^{-12} \mathrm{~W}$. Actually, it was found that the response of most junctions remained linear with input amplitude to levels of the order of the local oscillator amplitude, where the change in the $I-V$ characteristic due to the signal was clearly recorded. Hence, the dynamical range extended to the range of $10^{-9} \mathrm{~W}$. 
buting to conversion loss which do not affect the response to local oscillator amplitude only.

Eq. (6) is strictly valid only for the case that all signal power is converted into the difference frequency and no power flow was generated at other mixing frequencies. While possible in principle, this situation seldom occurs in practice. Extensive literature on conversion with resistive mixers (local oscillator driven semiconductor diodes) bear this out [15]. Because for the frequencies under consideration here $\left(\omega \ll \omega_{\mathrm{c}}\right)$ the junction performs essentially as switch of infinite conductance, periodically driven to some finite conductance by the local oscillator. Mixer theory developed for resistive mixers appears applicable. Accordingly the ideal limit $C=1$ for the resistive mixer is approached only under two conditions. The first condition is the requirement that currents at mixing frequencies other than the desired one (here $\omega-\omega_{\mathrm{LO}}$ ) are open or short circuited in order to avoid power-dissipation. This condition was certainly not obtained in our experiment because $\omega-\omega_{\text {LO }} \ll \omega_{\text {LO }}$ which brings the other sideband of the local oscillator at $2 \omega_{\text {LO }}-\omega$ into close proximity to $\omega$ and makes filtering of these frequencies extremely difficult. Assuming no other power flow except for the upper sideband immediately reduces the ideal limit to $C=0.5$, where the incoming signal power is equally divided into power at $\omega-\omega_{\text {LO }}$ and $2 \omega_{\text {LO }}-\omega$. Furthermore, since the impedance of weak links is relatively low compared to that of semiconductor diodes, the unavoidable contact capacitance is presumably less effective in short circuiting higher frequency components. Therefore, the remaining loss factor $(0.8$ for our best observed conversion ratio, $C_{\text {eff }}$ ) may have resulted from power flow at other mixing frequencies.

Our experiments, however, might also have fallen short in fulfilling the other requirement for obtaining the ideal conversion ratio. It is related to the harmonic content of the local oscillator amplitude generated by the junction nonlinearity. As was shown by Barber [16] (among others), the ideal limit is obtained under the condition that the first and second Fourier coefficient in the expansion of the periodic conductance variation relative to the average conductance approaches one. The condition is obtained if the conductance variation consists of short periodic pulses. For an ideal, resistive semi-conductor junction with an exponential characteristic the condition may be approached by proper bias and sufficient increase of oscillator amplitude. The non-monotonic behavior of Josephson junctions may prevent the attainment of the optimum switching condition, which could thus be a principle cause for not reaching the ideal conversion limit. The high efficiency with which point contacts could be used as a multiplier for the local oscillator frequency, however, appears to indicate a very rich harmonic spectrum (see Appendix 2) and, therefore, the argument may be invalid. Further numerical studies of the junction model should be able to answer this question.

4.4 NoISE. - The noise power spectral density in the limit $\hbar \omega \ll \mathrm{e}\langle V\rangle$ for highly damped junctions was found by Stephen and Scalapino [5], [6] to be

$$
\begin{array}{ll}
<i_{\mathrm{N}}^{2}>\rightarrow \frac{4 k T I_{\mathrm{B}}}{\langle V\rangle} & <V>\lesssim \frac{k T}{\mathrm{e}} \\
<i_{\mathrm{N}}^{2}>\rightarrow 2(n \mathrm{e}+p 2 \mathrm{e}) I_{\mathrm{B}} & <V>>\frac{k T}{\mathrm{e}}
\end{array}
$$

where $n$ and $p$ weigh the relative contributions of pairs and quasi particles, respectively. A spectrum of this form was observed experimentally on point contacts [17], [18]. Since here $\omega-\omega_{\text {LO }} \ll \mathrm{e}\langle V\rangle / \hbar$ and $\langle V\rangle \ll k T / \mathrm{e}(\approx 360 \mu \mathrm{V})$ the noise power output expected on the basis of eq. (7) would be

$$
P_{\mathrm{N}}=R_{\mathrm{D}} k T \frac{\Delta \mathrm{B} I_{\mathrm{B}}}{\langle V\rangle}=k T_{0} \Delta \mathrm{B}
$$

where $\Delta \mathrm{B}$ is the amplifier bandwidth. Note that generally $I_{\mathrm{B}} /\langle V\rangle>R_{\mathrm{J}}^{-1}$ or the noise contribution is in excess of that due to purely thermal fluctuations in $R_{\mathrm{J}}$. Inserting typical values $I_{\mathrm{B}} \approx 2 \times 10^{-5} \mathrm{~A}$, $R_{\mathrm{D}}=50 \Omega$ and $\langle V\rangle \approx 5 \times 10^{-5} \mathrm{~V}$ we find a noise equivalent temperature $T_{0} \approx 80 \mathrm{~K}$ of the order observed in the experiment $\left(50<T_{0}<190 \mathrm{~K}\right)$. Eq. (8) is unsatisfactory, however, in that by choosing the proper bias voltage, $\langle V\rangle$, any « excess » can be obtained. The bias dependence suggested by eq. (8) has not been observed in these experiments. No variation of noise with $\langle V\rangle$ could be tested on any one sample because of the matching requirement for $R_{\mathrm{D}}$ which fixed $\langle V\rangle$ and no systematic trend between different samples with differing $\langle V\rangle$ was observed. Furthermore, a reduction of $T_{0}$ with the application of local oscillator power, to be expected because of the reduction of $I_{\mathrm{B}}$ in eq. (8), was never observed. Therefore, it appears more adequate to rewrite eq. (8)

$$
P_{\mathrm{N}}=k T \in \frac{\Delta \mathrm{B} R_{\mathrm{D}}}{R_{\mathrm{J}}}
$$

where $\epsilon$ refers to the " excess » junction noise over purely thermal fluctuations whose detailed parameter dependence remains to be investigated.

The application of (noise free) local oscillator power transforms additional noise from the input into the difference frequency channel. If we assume a flat noise spectrum extending to frequencies in excess of $\omega$ one expects the output noise density to double for the ideal case with a conversion ratio $C=1$. In case both sidebands of the local oscillator frequency participate in power exchange with an ideal $C=0.5$, the result is the same, each sideband contributing just $1 / 2$ the noise power density. Considering double sideband 
operation the noise output power for arbitrary $C$ becomes $\left({ }^{4}\right)$.

$$
P_{\mathrm{N}}=k T \in \Delta \mathrm{B}(1+2 C) \frac{R_{\mathrm{D}}}{R_{\mathrm{J}}} .
$$

On our quietest junction (\# 11 of Table I) the increase in noise by application of local oscillator power was $T_{\mathrm{LO}} / T_{0}=1.4$ which with eq. (10) is roughly consistent with the assumption of a flat noise spectrum and effective conversion ratio $C_{\text {eff }} \approx 0.3$ which would result in $T_{\mathrm{LO}} / T_{0}=1.6$. $\left(C_{\mathrm{eff}} \approx 0.3\right.$ was measured for sample \# 18 with the lowest conversion loss, but could not be measured for sample \# 11.) It furthermore implies that the factor $S^{2}$ in eq. (6) cannot have differed greatly from one. It must be pointed out, however, that the application of local oscillator power required a readjustment of bias $\langle V\rangle$, generally to larger values, which could have affected the noise output. The experiments showed that once the junction noise exceeded several hundreds of degrees, no further increase of noise due to local oscillator power application was observed. The relative high noise temperatures, therefore, were probably caused by poor contact quality. The rather consistent results for the lowest measured noise temperatures, however, appear to indicate the order of noise temperatures which can be expected by junctions of this type.

4.5 COMPARISON WITH CONVENTIONAL MIXER DIODE. - Metal semi-conductor junctions or Schottky barrier diodes are the most commonly employed in the millimeter wave region because of their small capacity. Without going into a detailed description of resistive mixer operation it can be stated briefly that resistive mixers (i) exhibit a monotonic $I-V$ characteristic representing a conductance dependent on the local oscillator power over a range usually sufficient to approach good harmonic content required for ideal conversion performance. (ii) Power can be brought to the variable conductance only via an $\mathrm{RC}$ filter consisting of the contact capacitance and a series resistance due to the spreading of the current paths in the vicinity of the barrier region. This is the principle frequency limit encountered by resistive diodes. Frequency cutoff values of order $1000 \mathrm{GHz}$ and more have been reported [19]. (iii) Several sources add to noise in semiconductor diodes. Besides thermal and shot noise, generation-recombination and intervalley scattering can contribute. We quote the experimental results of noise measurements on cooled mixer diodes reported recently by Weinreb and Kerr [4]. These authors found noise temperatures of diodes without

(4) From eq. (10) the noise equivalent signal power follows with

$$
\mathrm{NEP}=k T \in \frac{2 C+1}{C} \frac{R_{\mathrm{D}}}{R_{\mathrm{J}}} .
$$

Clearly, the NEP is nearly optimized by $C \sim 1$. The improvement gained by $C>1$ is insignificant. local oscillator applied as low as $28 \mathrm{~K}$ (the physical temperature was $15 \mathrm{~K}$ ) and with local oscillator power applied the lowest measured noise temperature was $86 \mathrm{~K}$.

Comparing the measured performance of Josephson point contacts with that of resistive mixers we note that the generated noise is of comparable magnitude. The noise properties of Josephson junctions are not fully understood as yet, and, thus, no theoretically expected lower limit can be presently stated. It appears clear from experiment that Josephson junctions are not inferior to semiconductor diodes in their noise properties.

According to eq. (6) for certain parameter values conversion with Josephson junctions can be equal or better than that of resistive diodes. With respect to conversion properties the experiment indicates that losses beyond those predicted by eq. (6) occur, presumably because of mixing with higher harmonics. Since Josephson junctions have a rich spectrum of harmonics, this might be a serious disadvantage for the use of these junctions in the broadband application, emphasized in this work as well as high frequency diode work. This problem might only be overcome by the use of either contact capacitance or other means of frequency selectivity for which analysis becomes difficult. Beyond this drawback the simple junction model discussed here predicts a frequency cutoff due to pair phase modulation which is of the same order as that of modern resistive mixers. The inclusion of capacitive shunting and quasiparticle interference may further reduce this frequency cutoff, although it is expected that capacitance is less effective because of the low junction impedance $\left({ }^{5}\right)$.

5. Conclusion. - The above indicates that at this stage Josephson junctions used as passive elements for broadband mixer applications although comparable do not offer any advantage over resistive mixers. The biggest drawback for the application of Josephson junctions as mixers (as well as for any other function) is that they cannot be reproducibly manufactured, as yet, whereas resistive mixer production is based on a well-developed technology. It appears that the reproducible production of weak link or other Josephson type junctions with proper operating parameters is a prerequisite for (i) a more detailed investigation of the more subtle effects occurring in junctions (such as parametric effects), (ii) a successful application in millimeter wave technology. Under these circumstances passive Josephson junction mixers of the type described here might offer an interesting alternative to Schottky barrier mixers in special cases. For example, their multiplying property of the local oscillator

(5) The inclusion of capacitance into the circuit undoubtedly will lead to resonance effects either with $L$, which may result in better coupling efficiency and/or with the pair inductance, resulting in plasma effects. 
frequency could be used in case of frequency selective, narrow band operation. It is believed, however, that the real opportunity for Josephson junctions resides in their active, parametric function whose potential at high frequencies has not been explored as yet.

6. Appendix. - 6.1 Optimization OF INPUT NETWORK. - The coupling loss of the input network in the experiment for $S=1$ and $\alpha=1.4$ according to eq. (6), is limited to $C=0.063$. Here we briefly state the condition for which one can obtain $C \sim 1$. We summarize the boundary conditions for the wire impedance as stated in the text :a) $L \gtrsim 10 \hbar / 2 \mathrm{e} I_{\mathrm{c}}$ for the current driven resistive model to be valid ; $b) \omega L \gtrsim Z_{0}$ for $\alpha$ in eq. (3) to be $\alpha \gtrsim 1$; and $c$ ) $\omega L\rangle\left\langle Z_{\mathrm{J}}\right\rangle$ such that the actual form of $\left\langle Z_{\mathbf{J}}\right\rangle$ (inductive or resistive) is negligible in the calculation of $I_{\mathrm{LO}}$ and the junction not loaded by the remainder of the AC circuit. With $\omega L=Z_{0}$ eq. (6) reduced to $C=\alpha^{2} S^{2} R_{\mathrm{D}} / 4 \omega L$. Assuming again $\alpha^{2}=2$ and $S=0.9$ as reasonable $\left(\omega / \omega_{\mathrm{c}}=0.2\right)$ we obtain $C=0.4 R_{\mathrm{D}} / \omega L$. Since typically $R_{\mathrm{D}} \approx 5 R_{\mathrm{J}}$ and $L \approx 10 \hbar / 2 \mathrm{e} I_{\mathrm{c}}$ we find

$$
R_{\mathrm{D}} / \omega L=\omega_{\mathrm{c}} / 2 \omega
$$

or $R_{\mathrm{D}} / \omega L=2.5$ in our example. Thus, $C=1$. By choosing $R_{\mathrm{D}}=200 \Omega$ follows $\omega L=80 \Omega$, which because of $\omega L \approx Z_{0}$, requires a reduction in waveguide height to $1 / 5$. With, for example a $\mathrm{NbN}_{2}$ weak link, $\omega / 2 \pi=200 \mathrm{GHz}$. Conversion with $C>1$ is obtainable at smaller frequencies. It appears that the type of input network discussed here, which includes the unavoidable leads to the junction, is easily realizable in practice even in small waveguide sizes.

6.2 MIXING WITH THIRD HARMONIC OF LOCAL OSCILLATOR FREQUENCY. - An experiment was performed where local oscillator power at about $32 \mathrm{GHz}$ was fed to the junction and the change in conversion ratio observed compared to $95 \mathrm{GHz}$ operation. The local oscillator power was brought to the junction via a small diameter coaxial line, whose center conductor extended about $0.5 \mathrm{~mm}$ into the waveguide within a distance of $3 \mathrm{~mm}$ from the junction. The conversion ratio due to mixing with the third harmonic of the local oscillator frequency was reduced to $1 / 2$ of mixing with the fundamental. The relatively moderate reduction is considerably smaller than would be expected in a resistive mixer, where it has been shown that power of the $n$th harmonic decreases with $n^{-2}$ [20].

\section{References}

[1] Auracher, F., Dissertation, Dept of Electrical Engineering, UC Berkeley 1973.

[2] Hamilton, C. A., J. Appl. Phys. 44 (1973) 2371.

[3] Kanter, H. and Vernon Jr., F. L., J. Appl. Phys. 43 (1972) 3174.

[4] Weinreb, S. and Kerr, A. R., IEEE J. Solid State Circuits SC 8 (1973) 58.

[5] Stephen, M. T., Phys. Rev. 182 (1969) 531.

[6] Scalapino, D. T., University of Virginia Report No. AD 661848, 1967 (unpublished), PG-1. Available from Clearing House for Federal Scientific and Technical Information, Springfield, VA 22151.

[7] Aslamazov, L. G. and Larkin, A. I., Zh. Eksp. Teor. Fiz., Pisma' Red. 9 (1968) 150 ; JETP Lett. 9 (1969) 87.

[8] McCumber, D. E., J. Appl. Phys. 39 (1969) 3113.

[9] Stewart, W. C., Appl. Phys. Lett. 12 (1969) 277.

[10] Russer, P., J. Appl. Phys. 43 (1972) 2008.

[11] Likharev, K. K. and Semenov, V. K., Radiotekh. Elektron. 16 (1971) 2167.
[12] Zimmerman, J. E., Applied Superconductivity Conference, IEEE Conference Record 72 CHO 682-5-TABSC (1972) 544.

[13] McDonald, D. G., Private communication.

[14] Auracher, F. and van Duzer, T., J. Appl. Phys. 44 (1973) 848.

[15] Anand, Y. and Moroney, W. T., Proc. IEEE 59 (1971) 1182. ElDER, H. E. and Glinski, V. T., Microwave Semiconductor Devices and Their Circuit Applications, ed. Watson, H. A. (McGraw-Hill. Inc., New York) 1969, p. 370.

[16] BARber, M. R., IEEE Trans. Microwave Theory and Techniques MTT 15 (1967) 629.

[17] Kanter, H. and Vernon Jr., F. L., Appl. Phys. Lett. 16 (1970) 115 and Phys. Rev. Lett. 25 (1970) 588.

[18] Vernet, G. and Adde, R., Appl. Phys. Lett. 19 (1971) 195.

[19] Stover, H. L., Leedy, H. M. and Morehead, H. G., The Microwave Journal 16 (Feb. 1973) 35.

[20] Page, C. H., Proc. IRE 46 (1958) 1738. 